\title{
BMJ Open Prediabetes in pregnancy, can early intervention improve outcomes? A feasibility study for a parallel randomised clinical trial
}

\author{
Ruth C E Hughes, ${ }^{1}$ Janet Rowan, ${ }^{2}$ Jonathan Williman ${ }^{3}$
}

To cite: Hughes RCE, Rowan J, Williman J. Prediabetes in pregnancy, can early intervention improve outcomes? A feasibility study for a parallel randomised clinical trial. BMJ Open 2018;8:e018493. doi:10.1136/ bmjopen-2017-018493

- Prepublication history for this paper is available online. To view these files, please visit the journal online (http://dx.doi org/10.1136/bmjopen-2017018493).

Received 4 July 2017

Revised 12 December 2017 Accepted 29 January 2018
Check for updates

${ }^{1}$ Department of Obstetrics and Gynaecology, University of Otago, Christchurch, New Zealand

${ }^{2}$ Department of Obstetrics, National Women's Health, Auckland, New Zealand ${ }^{3}$ Biostatistics and Computational Biology Unit, University of Otago, Christchurch, New Zealand

Correspondence to

Dr Ruth C E Hughes;

ruth.hughes@cdhb.health.nz

\section{ABSTRACT}

Objective Measurement of glycated haemoglobin ( $\mathrm{HbA1C})$ in early pregnancy is routine in New Zealand to identify women with diabetes and prediabetes. However, the benefit of early intervention in women with prediabetes is inconclusive. Our aim was to test the feasibility of a twoarm parallel randomised controlled trial of standard care versus early intervention in pregnancies complicated by prediabetes.

Setting Two tertiary referral centres in New Zealand. Participants Women $<14$ weeks' gestation and HbA1C $\geq 5.9 \%-6.4 \%$ (41-46 mmol$/ \mathrm{mol})$ measured at booking, without pre-existing diabetes.

Interventions Randomisation was done by remote webbased allocation into one of two groups. Women in the early intervention group attended an antenatal diabetes clinic, commenced daily home blood glucose monitoring, and medication was prescribed if lifestyle measures failed to maintain target blood glucose levels. Controls received lifestyle education, continued standard care with their midwife and/or obstetrician, and were asked to perform a $75 \mathrm{~g}$ oral glucose tolerance test at 24 weeks' gestation with a referral to clinic if this test was positive. Both groups received lifestyle questionnaires at recruitment and in late pregnancy.

Outcome measures Recruitment rate, adherence to protocol and validation of potential primary outcomes. Results Recruitment rates were lower than expected, especially in Māori and Pacific women. Non-adherence to allocated treatment protocol was significant, $42 \%$ (95\% $\mathrm{Cl} 24 \%$ to $61 \%$ ) in the early intervention group and $30 \%$ $(95 \% \mathrm{Cl} 16 \%$ to $51 \%)$ in controls. Caesarean section and pre-eclampsia were signalled as potential primary outcomes, due to both the high observed incidence in the control group and ease of measurement.

Conclusions For a future definitive trial, extending the gestation of eligibility and stepped-wedge cluster randomisation may overcome the identified feasibility issues. Consistent with published observational data, pre-eclampsia and emergency caesarean section could be included as primary outcome measures, both of which have a significant impact on maternal and neonatal morbidity and healthcare costs.

Trial registration number ACTRN12615000904572; Preresults.

\section{Strengths and limitations of this study}

- Data to guide the antenatal management of women with prediabetes are lacking. This feasibility study is the first randomised controlled trial to investigate standard care versus early intervention in pregnancies complicated by prediabetes.

- This was multicentre study across two remote sites in New Zealand that included women from diverse ethnic backgrounds.

- Study numbers were low due to a suboptimal recruitment rate, particularly in M囚ori and Pacific women.

- The findings and conclusions are based on a small sample size and may not be generalisable.

\section{INTRODUCTION}

Glycated haemoglobin (HbAlc) is endorsed as an antenatal screening test for unrecognised type 2 diabetes in early pregnancy by several authorities. ${ }^{1}$ In New Zealand (NZ), HbA1c is measured routinely with the first-antenatal blood screen performed at booking. The 2014 NZ Ministry of Health Clinical Practice Gestational Diabetes (GDM) guideline $^{2}$ recommends that pregnant women with a $\mathrm{HbAlc}$ level $\geq 6.7 \%(50 \mathrm{mmol} / \mathrm{mol})$ at booking are referred immediately to an antenatal diabetes clinic for management, whereas women with a HbA1c level in the prediabetes range of $5.9 \%-6.6 \% \quad(41-49 \mathrm{mmol} /$ mol) receive early lifestyle advice, including diet and weight gain recommendations, and a glucose tolerance test (GTT) at 24 weeks' gestation.

Women identified with prediabetes in early pregnancy have been reported to have an increased risk of adverse pregnancy outcomes compared with women with lower HbAlc levels at booking. ${ }^{3}$ However, it is not clear whether pregnancy outcomes can be improved by managing women with prediabetes through diabetes clinics from early pregnancy versus 
screening them for GDM in later pregnancy. Currently, both management approaches are practised within NZ, with concern from some clinicians that the NZ HbAlc cut-point of $6.7 \%(50 \mathrm{mmol} / \mathrm{mol})$ for early referral is set too high. The NZ GDM guideline acknowledges that data are lacking in this area and that an evidence-based recommendation cannot be made until high-quality evidence becomes available to guide consistent care. A recent observational study of pregnant women without pre-existing diabetes compared three groups: those with elevated HbA1c (5.9\%-6.6\%, 41-49 $\mathrm{mmol} / \mathrm{mol})$ referred to a diabetes clinic before 24 weeks' gestation (early treatment), those with elevated HbAlc at diagnosis of GDM referred to diabetes clinic after 24 weeks' gestation (late treatment) and those with a HbAlc $<5.9 \%$ (41 mmol/ mol) at diagnosis of GDM referred to diabetes clinic after 24 weeks' gestation (routine GDM). ${ }^{4}$ The early treatment and routine GDM groups had similar outcomes, whereas the late-treatment group had worse outcomes, compared with the routine GDM group, with increased rates of pre-eclampsia, preterm birth, birth weight $>4000 \mathrm{~g}$ and neonatal admission. Pre-eclampsia and preterm birth may be associated with placental function and interventions to improve placental function may show greater efficacy if commenced before 16 weeks' gestation, as shown with aspirin. ${ }^{5}$

Our aim was to assess the feasibility of a parallel randomised controlled trial (RCT) of early intervention versus standard care (controls) in pregnant women with a HbAlc measurement of 5.9\%-6.4\% (41-46 mmol/mol) in early pregnancy. Potential feasibility issues identified a priori were achieving a sustainable recruitment rate in early pregnancy and compliance with the intervention. We also evaluated the feasibility of using pre-eclampsia and preterm delivery as potential primary outcome measures in a future definitive RCT.

\section{METHODS}

\section{Setting}

We conducted a multicentre, randomised controlled, two-arm, parallel-group, unblinded feasibility trial with 1:1 randomisation. Two tertiary referral centres, Christchurch Women's Hospital and National Women's Hospital Auckland, took part in the trial and participant randomisation was stratified by site using a web-based randomisation service. ${ }^{6}$ Inclusion criteria were a HbAlc level of $5.9 \%-6.4 \%(41-46 \mathrm{mmol} / \mathrm{mol})$ measured at booking, an ongoing pregnancy with gestational age $<14$ weeks' gestation and a minimum maternal age of 18 years. Exclusion criteria were pre-existing overt diabetes, a fetus with a lethal congenital anomaly and multiple pregnancy.

\section{Participants}

Eligible women were identified by clinical staff, who scanned the referral letters to antenatal diabetes clinic from lead maternity carers (community midwife or obstetrician) and general practitioners. In Christchurch, a laboratory alert appears on the blood test result when an antenatal HbAlc measurement is $\geq 5.9 \%$ (41 mmol/ mol) to contact the local diabetes in pregnancy service for management advice. In Auckland, community midwives were educated and encouraged to refer women to antenatal diabetes clinic if they had an elevated HbA1c $\geq 5.9 \%(41 \mathrm{mmol} / \mathrm{mol})$ at booking. All referred women were offered a lifestyle education session with their local antenatal diabetes education team, including standard weight gain and dietary advice. ${ }^{7}$ All eligible women referred to clinic were approached with the study information. Interested participants were followed up by a researcher, in person or by phone, to arrange a time to obtain informed written consent. A list of women who declined to participate was kept, as ethical approval was also granted to collect audit data from their hospital records.

\section{Intervention}

Women consenting to the study were randomised into one of two groups. The early intervention group were offered outpatient visits every 3-6 weeks throughout pregnancy (frequency determined by the attending physician) at their local antenatal diabetes clinic, in combination with follow-up with their lead maternity carer (community midwife or obstetrician). They received ongoing lifestyle education, commenced home blood glucose (BG) monitoring using a CareSens N-automated glucometer to read capillary BG measurements both before and after each meal, and commenced medication as required (metformin and/orinsulin as advised by the attending physician) to maintain capillary BG levels within the usual targets for pregnancy: fasting $\mathrm{BG}<5.0 \mathrm{mmol} / \mathrm{L}(90 \mathrm{mg} /$ $\mathrm{dL})$, 1-hour postprandial BG $<7.4 \mathrm{mmol} / \mathrm{L}(133.3 \mathrm{mg} /$ $\mathrm{dL}$ ) and 2-hour postprandial $\mathrm{BG}<6.5 \mathrm{mmol} / \mathrm{L}(118 \mathrm{mg} /$ $\mathrm{dL})$. The controls received standard care with their lead maternity carer and were asked to take a $75 \mathrm{~g}$ oral GTT to screen for GDM at 24 weeks' gestation with appropriate follow-up if this test was positive. NZ GDM criteria were used to define a positive GTT, fasting BG $\geq 5.5 \mathrm{mmol} / \mathrm{L}$ $(99 \mathrm{mg} / \mathrm{dL})$ or 2 -hour BG $\geq 9.0 \mathrm{mmol} / \mathrm{L}(162 \mathrm{mg} / \mathrm{dL})$; although the International Association of Diabetes and Pregnancy Study Groups (IADPSG) criteria $^{8}$ are also reported. Both groups were asked to complete a diet, activity and well-being questionnaire at enrolment and at 36 weeks' gestation. The questionnaires were based on validated questionnaires, the WHO-5 Well-Being Index, ${ }^{9}$ the Global Physical Activity Questionnaire ${ }^{10}$ and the Food and Activity Questionnaire used during the metformin in gestational diabetes (MiG) trial. ${ }^{11}$ A WHO-5 score of 50 or below indicates low mood, and a score of 28 or below indicates likely depression and warrants further assessment. During this study, women with a WHO-5 score of 28 or below were referred to their general practitioners for follow-up.

\section{Outcome measures}

Feasibility issues identified a priori were the following: 
1. The ability to demonstrate a sustainable recruitment rate $<14$ weeks' gestation, particularly in women disadvantaged socially who have a high risk for diabetes but are less likely to book with a lead maternity carer in early pregnancy such as Māori and Pacific women. ${ }^{12} \mathrm{~A}$ sustainable recruitment rate was defined as $\geq 15$ women per month or as the recruitment and retention of at least $30 \%$ of eligible women. This was in order to attain target participant numbers within the time constraints of a future definitive 3-year trial.

2. To achieve a high level of participant compliance with the intervention, defined as $\geq 80 \%$. A non-compliance rate of greater than $20 \%$ would make the sample size for a future definitive trail difficult to achieve. Compliance was defined as the ability of participants to comply with their allocated treatment plan. Compliance in the early intervention group was measured by rates of home $\mathrm{BG}$ monitoring, clinic attendance rates, compliance with medication, withdrawing from the study and/or performing a GTT and modifying their treatment plan based on the results. Compliance in the control group was measured by abstaining from BG measurements (GTT or home BG monitoring) before 24 weeks' gestation and by completion of a GTT at $\geq 24$ weeks' gestation with appropriate follow-up as required.

3. To develop a suitable primary outcome for the definitive study. Potential primary outcomes were defined as being clinically relevant outcomes that are potentially sensitive to the intervention and which can be measured precisely across sites. Based on earlier studies, pre-eclampsia and preterm birth were outcomes of interest, caesarean section, birth weight and neonatal morbidity were also explored. In both groups, changes to dietary intake and activity levels were assessed by the diet and activity questionnaire, and potential harm resulting from the interventions was assessed using a patient well-being score. Pre-eclampsia was defined by the International Society for the Study of Hypertension in Pregnancy criteria as new-onset or worsening hypertension after 20 weeks' gestation and the coexistence of one or more of the following new-onset conditions: proteinuria (protein/creatinine ratio $30 \mathrm{mg} / \mathrm{mmol}$ ), other maternal organ dysfunction or fetal growth restriction. Perinatal deaths were defined by NZ criteria as deaths occurring $\geq 20$ weeks' gestation or at a birth weight of $\geq 400 \mathrm{~g}$ irrespective of gestation, up to 28 completed days after birth. Shoulder dystocia was defined as a difficult delivery after birth of the head, requiring manoeuvres such as McRoberts manoeuvre, suprapubic pressure or intravaginal manipulation. Customised birthweight centiles were calculated using the gestation related optimal weight (GROW) Bulk Centile Calculator (V.6.7.8, 2017) from the Perinatal Institute, Birmingham, UK, which adjusts for maternal age, parity, height, weight (without limits) and ethnicity as well as for gestational age at delivery and sex.

\section{Statistical analysis}

A biostatistician was involved in study design and performed the analysis. As this was a feasibility study, a sample size calculation was not performed and the length of the study was decided on to allow a good estimate of the monthly recruitment rate. Based on both the birth rate (approximately 6000 per annum at each recruitment site) and on the percentage of women (approximately $2 \%-4 \%)$ that we expect to have a screening HbA1c level $5.9 \%-6.4 \%(41-46 \mathrm{mmol} / \mathrm{mol})$ measured at booking, we estimated to recruit 10-15 women per month or 100 women within the 8-month recruitment phase of the study. Allowing for a 15\% attrition rate from miscarriage, this would be provide a sample size of approximately 85 participants to inform us about the feasibility and practicalities of running a larger definitive trial. Study data were collected and managed using REDCap electronic data capture tools hosted at the University of Otago. ${ }^{13}$ Double entry of data was performed to check for accuracy. The feasibility outcomes were reported descriptively and narratively, continuous data as mean (IQR) and categorical outcomes as count (percentage). Baseline characteristics and pregnancy outcomes of randomised and non-randomised participants were summarised descriptively, but as this was a feasibility study inferential statistics (CIs and $\mathrm{P}$ values) testing the efficacy of treatment are not reported.

\section{RESULTS}

During the 8-month enrolment period from October 2015 to May 2016, 69 women without known diabetes and a HbAlc $5.9 \%-6.4 \%(41-46 \mathrm{mmol} / \mathrm{mol})$ measured at booking were referred to the two sites. Of the women referred, 67 were eligible for the study (figure 1).

Of the 67 women approached, 47 (70\%, 95\% CI 58\% to $80 \%$ ) consented to randomisation. The 20 women who declined to participate were managed according to standard care as were the control group. The feasibility study ended when the planned interval for conduct was reached.

Characteristics of the women who did and did not participate in the trial are shown in table 1. Numbers were too small to demonstrate significant differences between the groups. A greater proportion of Māori 3/5 $(60 \%)$ and Pacific 5/12 (42\%) women declined to participate compared with Europeans 3/11 (27\%) and Asians $7 / 34(21 \%), \mathrm{P}=0.66$ and $\mathrm{P}=0.64$, respectively. There was one pregnancy loss in each group, one at $13+4$ weeks' gestation in the early intervention group and one at $17+1$ weeks' gestation in the control group. GTTs were performed by $21 / 22(96 \%)$ of the remaining controls, $13 / 21(62 \%, 95 \%$ CI $41 \%$ to $79 \%)$ were abnormal by NZ GTT criteria and $15 / 21(71 \%, 95 \%$ CI $50 \%$ to $86 \%)$ by IADPSG criteria. BG-lowering medication was prescribed for $11 / 22(50 \%, 95 \%$ CI $31 \%$ to $69 \%)$ of the control group (metformin in 3 and insulin in 11 women) and 17/23 $(74 \%, 95 \%$ CI $54 \%$ to $87 \%)$ of the early intervention 


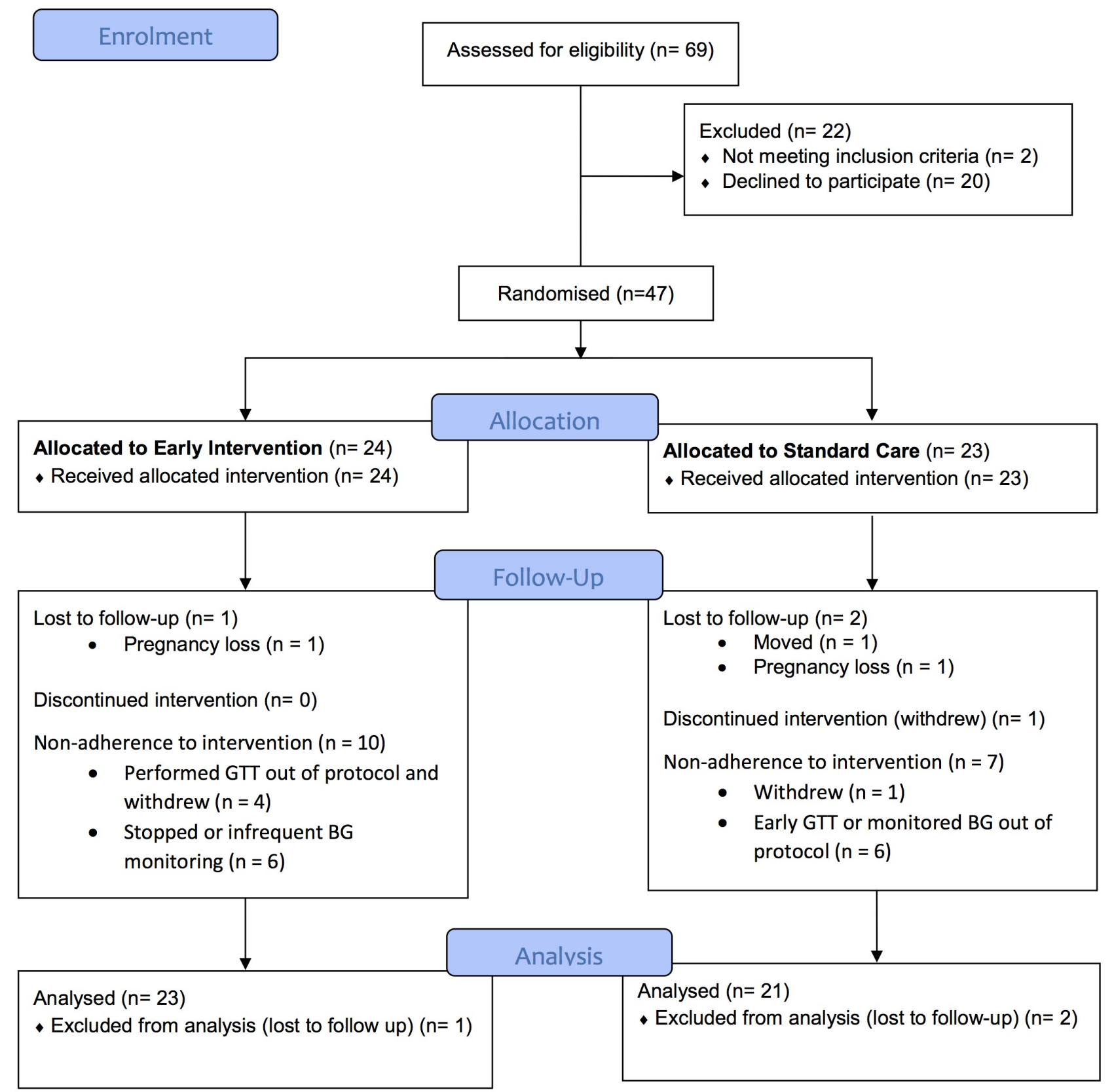

Figure 1 Flow diagram of recruitment and randomisation. BG, blood glucose; GTT, $75 \mathrm{~g}$ oral glucose tolerance test.

group (metformin in 14 and insulin in 15 women). In the early intervention group, $17 / 17(100 \%, 95 \%$ CI $81 \%$ to $100 \%$ ) of the prescriptions for BG-lowering medication were made before 24 weeks' gestation and 15/17 ( $88 \%, 95 \%$ CI $66 \%$ to $97 \%$ ) before 20 weeks' gestation. In women who declined to be randomised, 16/20 (80\%) performed a GTT, of which $7 / 16(45 \%, 95 \%$ CI $23 \%$ to $67 \%)$ were positive for GDM by NZ GTT criteria, $9 / 16$ (56\%, 95\% CI $33 \%$ to $77 \%$ ) by IADPSG criteria; all 7 women referred with a diagnosis of GDM were prescribed BG-lowering medications (metformin in 3, insulin in 7).
Fewer women were referred to clinic than predicted with a mean (SD) 5.9 (7.0) women recruited per calendar month. A sharp fall in the recruitment rate in Auckland from $\geq 14$ women per month to $0-6$ per month, took place after a change in the HbAlc assay 3 months into the study. The assay was changed from the Roche Cobas Tina-Quant HbA1c Gen.2 assay performed on a Roche Integra 800 instrument to the Roche Cobas Tina-Quant HbA1c Gen.3 assay performed on a Cobas C513 instrument, with the new HbAlc assay reading $0.24 \%(3 \mathrm{mmol} / \mathrm{mol}$ ) lower but in line with assays at other centres throughout NZ. 
Table 1 Maternal characteristics

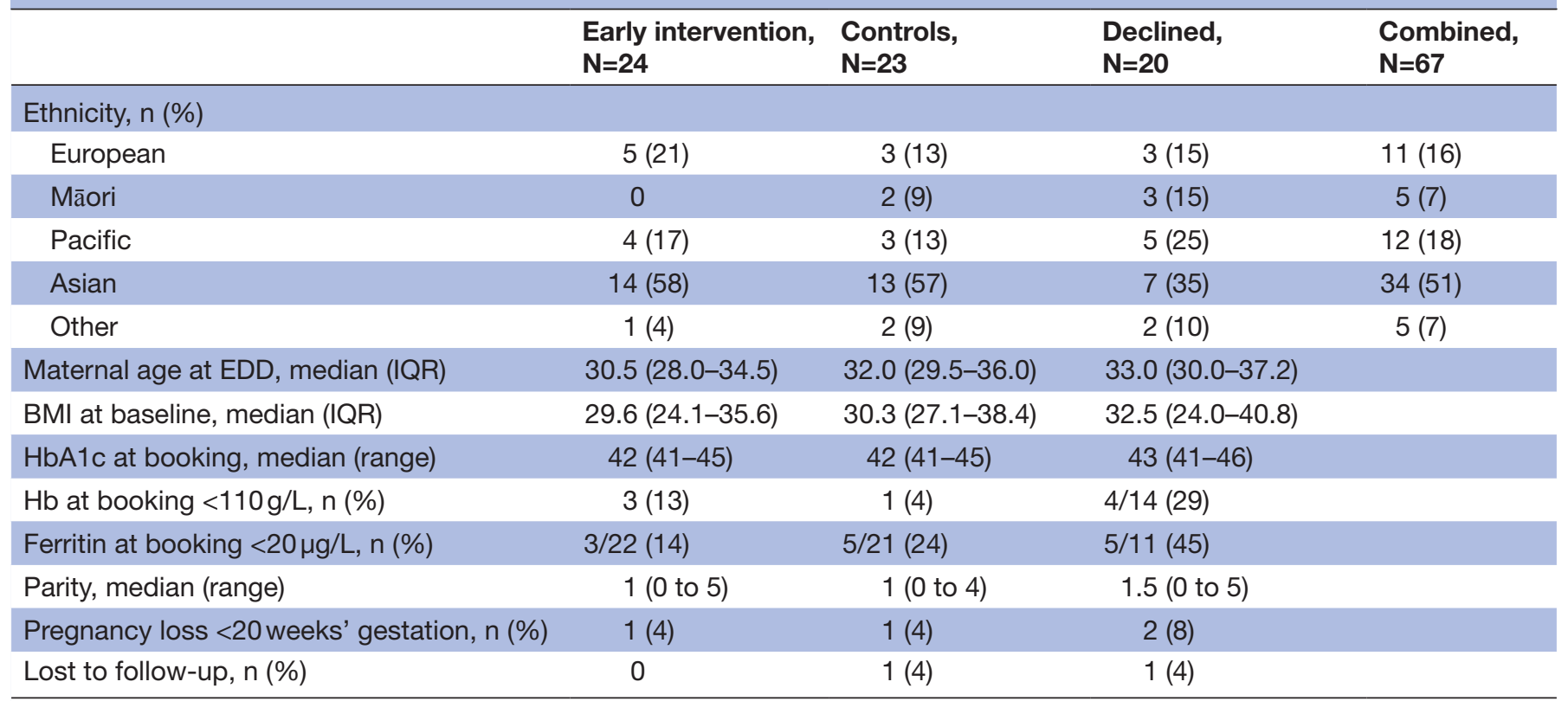

BMI, body mass index; EDD, expected date of delivery; Hb, haemoglobin; HbA1c, glycated haemoglobin.

After the first 3 months of the trial, a sustainable recruitment before 14 weeks' gestation was deemed unfeasible. We then moved to recruiting women once a pregnancy ultrasound scan had confirmed fetal viability and the gestational age at recruitment was extended to $<16$ weeks' in Auckland and <20 weeks' in Christchurch, which improved recruitment and retention rates by capturing women who booked later in pregnancy and the late/ delayed referrals.

Participant compliance with the allocated intervention was suboptimal in both groups, with a deviation from protocol in $42 \%(10 / 23) 95 \%$ CI $24 \%$ to $61 \%$ of the early intervention group and in $30 \%(7 / 24) 95 \% \mathrm{CI}$ $16 \%$ to $51 \%$ of controls. In the early intervention group, a number of women who were non-adherent with recommendations commented that the study information and consent form for the trial had reassured them that no treatment was necessary before 24 weeks' gestation: $6 / 23(25 \%)$ ceased or infrequently measured their BG levels; 11/23 (46\%) were non-compliant with BG-lowering medication when recommended; 9/23 (38\%) did not attend outpatient clinic appointments as arranged. In the control group, a number of women had been treated in a previous pregnancy or their maternity caregiver was concerned they were not receiving treatment (which had been standard in Auckland before the trial): 2/24 (9\%) performed home BG monitoring early without undergoing a GTT; 4/24 (17\%) performed an early GTT before the protocol timing of 24 weeks' gestation; 2/24 (9\%) were non-adherent with BG- lowering medication when recommended and 1/24 (4\%) did not attend outpatient clinic appointments as arranged. One $(4 \%)$ woman from the control group withdrew from the study as she moved location, whereas $4 / 23(17 \%)$ women in the early intervention group withdrew from the study and in each case this followed completion of an 'off-protocol' GTT with a normal result.

Pregnancy outcomes of potential interest as outcome measures for a larger definitive study are described in table 2. Pre-eclampsia and emergency caesarean section are both clinically relevant outcomes that were easy to measure across centres and they occurred at a reasonable frequency to be considered as primary outcomes. Preterm birth and customised birth weight were both easy to measure, but neither occurred at a higher frequency than expected.

Questionnaire responses are reported in table 3. After excluding women who were lost to follow-up or who withdrew from the study, the late-pregnancy questionnaire was completed by $74 \%(14 / 19), 95 \%$ CI $51 \%$ to $88 \%$, of the early intervention group and $90 \%(18 / 20), 95 \%$ CI $70 \%$ to $97 \%$, of controls. At randomisation, $11 \%(5 / 46)$, $95 \%$ CI $5 \%$ to $23 \%$, of recruits had a WHO-5 score indicative of depression that required further assessment.

\section{DISCUSSION}

We assessed the feasibility of a RCT of standard care versus early intervention in pregnant women with a HbAlc within the prediabetes range measured at booking. This study confirmed that the potential feasibility issues identified a priori were valid. In particular, we did not demonstrate a sustainable recruitment rate and participant compliance was suboptimal at $<60 \%$ in the early intervention group and $<70 \%$ in the controls. Pre-eclampsia and emergency caesarean section were signalled as potentially suitable outcome measures.

The strength of this study is that it was conducted across two geographically distant centres in NZ, and thus the feasibility of conducting a multicentre study 
Table 2 Pregnancy outcomes excluding pregnancies with early fetal loss or lost to follow-up

\begin{tabular}{|c|c|c|c|}
\hline & $\begin{array}{l}\text { Early intervention, } \\
\mathrm{N}=23\end{array}$ & $\begin{array}{l}\text { Controls, } \\
\mathrm{N}=21\end{array}$ & $\begin{array}{l}\text { Declined, } \\
\mathrm{N}=17\end{array}$ \\
\hline \multicolumn{4}{|l|}{ Ultrasound scan-fetal measurements, $n(\%)$} \\
\hline Abdominal circumference $>95$ th centile & 0 & $1(5)$ & $6(35)$ \\
\hline Abdominal circumference $<5$ th centile & 0 & $3(14)$ & $1(6)$ \\
\hline Estimated fetal weight $>90$ th centile & $3(13)$ & $5(24)$ & $7(41)$ \\
\hline Estimated fetal weight $<10$ th centile & $1(4)$ & $3(14)$ & $1(6)$ \\
\hline Pre-eclampsia, n (\%) & 0 & $3(14)$ & $1(6)$ \\
\hline Induction of labour, $\mathrm{n}(\%)$ & $10(43)$ & $11(52)$ & $3(18)$ \\
\hline \multicolumn{4}{|l|}{ Caesarean section, $\mathrm{n}(\%)$} \\
\hline Total & $6(26)$ & $9(43)$ & $11(65)$ \\
\hline Elective & $2(9)$ & $3(14)$ & \\
\hline Emergency & $4(17)$ & $6(29)$ & \\
\hline Gestation at delivery $<37$ weeks, $n(\%)$ & $1(4)$ & $1(5)$ & $1(6)$ \\
\hline Shoulder dystocia, birth trauma, neonatal death, $\mathrm{n}(\%)$ & 0 & 0 & 0 \\
\hline Customised birthweight centile, median (IQR) & $49(21-72)$ & $37(20-57)$ & $77(32-97)$ \\
\hline Customised birthweight centile <10th, $n(\%)$ & $4(17)$ & $4(19)$ & $3(18)$ \\
\hline Customised birthweight centile >90th, n (\%) & $1(4)$ & $2(10)$ & $4(24)$ \\
\hline Baby BGL checked, $\mathrm{n}(\%)$ & $19(83)$ & $16(76)$ & $10(59)$ \\
\hline Baby BSL $<2.6 \mathrm{mmol} / \mathrm{L}$ & $7 / 19(37)$ & 2/16 (13) & $7 / 10(70)$ \\
\hline Dextrose gel & $5 / 19(22)$ & $2 / 16(13)$ & $5 / 10(50)$ \\
\hline Intravenous dextrose & 0 & 2/16 (13) & $2 / 10(20)$ \\
\hline NICU admission, $\mathrm{n}(\%)$ & $1(4)$ & $2(10)$ & $2(10)$ \\
\hline Respiratory support >4 hours, $\mathrm{n}(\%)$ & 0 & $2(10)$ & 0 \\
\hline Jaundice requiring phototherapy, n (\%) & $1(4)$ & 0 & $1(5)$ \\
\hline
\end{tabular}

BGL, blood glucose level; BSL, blood sugar level; NICU, neonatal intensive care unit.

Table 3 Results of the study questionnaires completed at recruitment and in late pregnancy (at approximately 36 weeks' gestation)

\begin{tabular}{|c|c|c|c|c|}
\hline Completed questionnaires & $\begin{array}{l}\text { Early intervention } \\
\text { at randomisation, } \\
\mathrm{N}=\mathbf{2 4 / 2 4}\end{array}$ & $\begin{array}{l}\text { Early intervention } \\
\text { late pregnancy, } \\
\mathrm{N}=14 / 19\end{array}$ & $\begin{array}{l}\text { Controls } \\
\text { at randomisation, } \\
\mathrm{N}=22 / 23\end{array}$ & $\begin{array}{l}\text { Controls } \\
\text { late pregnancy, } \\
\mathrm{N}=18 / 20\end{array}$ \\
\hline \multicolumn{5}{|l|}{ Activity questionnaire } \\
\hline $\begin{array}{l}10 \text { min of continuous exercise } \\
<3 \text { days/week }\end{array}$ & $11(46)$ & $4(29)$ & $10(45)$ & $4(22)$ \\
\hline No recreational exercise & $15(63)$ & $5(36)$ & $14(64)$ & $11(61)$ \\
\hline$>8$ hours/day of sedentary behaviour & $9(38)$ & $2(15)$ & $9(41)$ & $3(17)$ \\
\hline \multicolumn{5}{|l|}{ Food questionnaire } \\
\hline Juice $<1$ day/week & $15(63)$ & $10(71)$ & $12(55)$ & $12(67)$ \\
\hline Fizzy drink $<1$ day/week & $17(71)$ & $13(93)$ & $17(77)$ & $16(89)$ \\
\hline Take away <1 day/week & $14(58)$ & $7(50)$ & $9(41)$ & $8(44)$ \\
\hline Vegetables 7 days/week & $16(67)$ & $11(79)$ & $11(50)$ & $14(78)$ \\
\hline \multicolumn{5}{|l|}{ Well-Being questionnaire WHO-5 } \\
\hline Score $\leq 28$ & $1(4)$ & $1(7)$ & $4(18)$ & $1(6)$ \\
\hline Overall score mean (SD) & $68.00(17.01)$ & 73.33 (14.32) & $55.27(27.81)$ & $69.33(17.08)$ \\
\hline
\end{tabular}


and recruiting in ethnically diverse populations were assessed. The major limitation was the low recruitment rate, providing fewer data for interpretation that in turn could incorrectly guide us in planning a future definitive trail. The low numbers meant that some covariates were not well balanced between the treatment groups, but as the outcome measures of this study were related to feasibility and not to clinical outcomes the mismatch was less important. Although we conclude that the protocol of this feasibility study was not suitable for a definitive trial, we do not know with certainty that our recommend protocol changes (below) are feasible.

Factors influencing our recruitment rate included an unforeseen change in the HbA1c assay in Auckland, which greatly reduced the number of referrals of potentially eligible women. We predicted that $\sim 2 \%-4 \%$ of the birthing population would be eligible, whereas since the conclusion of this study, prospective laboratory data from Auckland indicate that only $\sim 1.2 \%$ are eligible. Further only $0.9 \%$ of the birthing population $(\sim 80 \%$ of potential recruits) were referred. Recruitment was also hampered by the limits placed on the gestation of eligibility. However, extending the gestation of eligibility to 20 weeks' may result in too small a difference between groups with respect to the onset and duration of the intervention, potentially masking any benefit of early intervention. An option for a definitive trial would be to extend the gestation of eligibility and perform subgroup analysis of outcomes by gestation at recruitment. Recruitment rates were particularly low in Māori and Pacific women. We assessed the characteristics of women who declined to participate to see if we captured a representative cohort of our population, as we want the results of a definitive study to be generalisable across NZ. Non-European women in NZ have a higher rate of prediabetes ${ }^{12}$ and Māori and Pacific women in particular have a greater risk of adverse pregnancy outcomes, thus, they may benefit the most from early intervention. ${ }^{14}$ Māori and Pacific women were the least likely to consent to this study and they are known to book at a later gestation than European women. ${ }^{12}$ These barriers may be overcome by both extending the gestation of eligibility and randomisation by site rather than at the individual level.

A high proportion of participants did not adhere to the assigned intervention. Non-compliance with BG monitoring may be less likely if randomisation is by site rather than at the individual level, which may be influenced by a participant's prior obstetric history, by their interpretation of the study information sheet that stated that both treatment options may be equally effective, or by the practice of the lead maternity carer. Compliance with medication is likely to be somewhat of an issue for both arms of the study irrespective of study design, highlighting the importance of positive relationships between patients and healthcare workers and adequate patient education and follow-up.

Both pre-eclampsia and caesarean section are clinically relevant outcomes that impact significantly on maternal and neonatal morbidity and on healthcare costs. ${ }^{15}$ The emergency caesarean section rate in the control arm of this study was higher than our national total caesarean section rate in NZ $(<20 \%)$. Logically, early intervention versus intervention commencing at 24 weeks' gestation is more likely to improve outcomes related to placentation, such as pre-eclampsia and preterm birth, whist both interventions may improve outcomes related to fetal hyperinsulinaemia, such as macrosomia and neonatal hypoglycaemia. Abnormal placentation is integral to the later development of pre-eclampsia and placentation largely occurs in the first trimester, which may explain why aspirin prophylaxis against pre-eclampsia is more effective when commenced before 16 weeks' gestation. ${ }^{5}$

\section{CONCLUSION}

A future definitive study exploring standard care versus early intervention, in pregnant women without known pre-existing diabetes and with a HbAlc $\geq 5.9 \%-6.4 \%$ $(41-46 \mathrm{mmol} / \mathrm{mol})$ measured at booking, is likely to be feasible with modifications to the study design. We propose a cluster step-wedge randomised study design across 10 sites in NZ. Randomisation by site rather than at the individual level would help reduce both the ethnic disparity in recruitment and the 'contamination' rate between the two intervention arms of the study.

Contributors RCEH contributed to the study design, data collection, analysis of data and preparation of the final document. JR contributed to the study design, data collection, analysis of data and preparation of the final document. JW contributed to the study design, analysis of data and preparation of the final document. All authors read and approved the final document.

Funding This study was supported by the Health Research Council of New Zealand (grant no: 15/401).

\section{Competing interests None declared.}

Patient consent Detail has been removed from this case description/these case descriptions to ensure anonymity. The editors and reviewers have seen the detailed information available and are satisfied that the information backs up the case the authors are making.

Ethics approval The study was approved by the New Zealand Health and Disability Ethics Committees (reference 15/NTA/98/AM03).

Provenance and peer review Not commissioned; externally peer reviewed. Data sharing statement № additional data are available.

Open Access This is an Open Access article distributed in accordance with the Creative Commons Attribution Non Commercial (CC BY-NC 4.0) license, which permits others to distribute, remix, adapt, build upon this work non-commercially, and license their derivative works on different terms, provided the original work is properly cited and the use is non-commercial. See: http://creativecommons.org/ licenses/by-nc/4.0/

(c) Article author(s) (or their employer(s) unless otherwise stated in the text of the article) 2018. All rights reserved. No commercial use is permitted unless otherwise expressly granted.

\section{REFERENCES}

1. American Diabetes Association. 2. Classification and diagnosis of diabetes. Diabetes Care 2017;40(Suppl 1):S11-24.

2. Ministry of Health. Screening, diagnosis and management of gestational diabetes in new zealand: a clinical practice guideline. Wellington: Ministry of Health, 2014. 
3. Hughes RC, Moore MP, Gullam JE, et al. An early pregnancy HbA1c $\geq 5.9 \%(41 \mathrm{mmol} / \mathrm{mol})$ is optimal for detecting diabetes and identifies women at increased risk of adverse pregnancy outcomes. Diabetes Care 2014;37:2953-9.

4. Rowan JA, Budden A, Ivanova V, et al. Women with an $\mathrm{HbA} 1 \mathrm{c}$ of $41-49 \mathrm{mmol} / \mathrm{mol}(5.9-6.6 \%)$ : a higher risk subgroup that may benefit from early pregnancy intervention. Diabet Med 2016;33:25-31.

5. Bujold E, Roberge S, Lacasse Y, et al. Prevention of preeclampsia and intrauterine growth restriction with aspirin started in early pregnancy: a meta-analysis. Obstet Gynecol 2010;116(Pt 1):402-14.

6. Sealed envelope. Randomisation and online databases for clinical trials. https://sealedenvelope.com

7. Ministry of Health. Guidance for healt guidancefor healthy weight gain during pregnancy. record card hy weight gain during pregnancy. 2014 www.health.govt.nz

8. Metzger BE, Gabbe SG, Persson B, et al. International association of diabetes and pregnancy study groups recommendations on the diagnosis and classification of hyperglycemia in pregnancy. Diabetes Care 2010;33:676-82.

9. Topp CW, Østergaard SD, Søndergaard S, et al. The WHO-5 Well-Being Index: a systematic review of the literature. Psychother Psychosom 2015;84:167-76.
10. World Health Organization. Global Physical Activity Questionnaire (GPAQ). 2017 www.who.int/chp/steps/resources/GPAQ_Analysis_ Guide.pdf

11. Rowan JA, Hague WM, Gao W, et al. Metformin versus insulin for the treatment of gestational diabetes. N Engl J Med 2008;358:2003-15.

12. Hughes RC, Williman J, Gullam JE. Universal HbA1c measurement in early pregnancy to detect type 2 diabetes reduces ethnic disparities in antenatal diabetes screening: a population-based observational study. PLoS One 2016;11:e0156926.

13. Harris PA, Taylor R, Thielke R, et al. Research electronic data capture (REDCap)-a metadata-driven methodology and workflow process for providing translational research informatics support. J Biomed Inform 2009;42:377-81.

14. Health Quality and Safety Commission New Zealand. Tenth annual report of the perinatal and maternal mortality review committee: reporting mortality 2014. Wellington: Health Quality and Safety Commission New Zealand, 2016.

15. Bennett WL, Robinson KA, Saldanha IJ, et al. High priority research needs for gestational diabetes mellitus. J Womens Health 2012;21:925-32. 